\title{
Formation Control for Multiple Quadrotor Aircraft via Fixed-time Consensus Algorithm
}

\author{
Xiaohua Zhang $\mathbb{D}^{1},{ }^{1}$ Junli Gao $\mathbb{D},{ }^{2}$ Wenfeng Zhang $\mathbb{D},{ }^{3}$ Tao Zeng, ${ }^{1}$ and Liping Ye ${ }^{1}$ \\ ${ }^{1}$ College of Automation, Zhongkai University of Agriculture and Engineering, 501 Zhongkai Road, Guangzhou 510225, China \\ ${ }^{2}$ School of Automation, Guangdong University of Technology, Guangzhou 510006, China \\ ${ }^{3}$ Institute of Rural Development, Zhongkai University of Agriculture and Engineering, 501 Zhongkai Road, \\ Guangzhou 510225, China \\ Correspondence should be addressed to Junli Gao; robotsys2000@163.com and Wenfeng Zhang; zhwf999@163.com
}

Received 2 June 2019; Revised 2 August 2019; Accepted 29 August 2019; Published 25 September 2019

Academic Editor: Ioannis Kostavelis

Copyright ( 2019 Xiaohua Zhang et al. This is an open access article distributed under the Creative Commons Attribution License, which permits unrestricted use, distribution, and reproduction in any medium, provided the original work is properly cited.

\begin{abstract}
This paper mainly studies the formation control problem of multiple quadrotor aircraft via fixed-time control theory. First, based on the bilimit homogeneous theory and the framework of multiagent theory, for multiaircraft, a fixed-time formation control strategy is proposed. Considering the external disturbance existing on the attitude loop of the aircraft, the corresponding fixedtime disturbance observer is designed with the observer technology. Then, a fixed-time attitude controller is designed based on the accurate observation and fast compensation from the disturbance observer. Finally, some simulations are performed to verify the effectiveness of the proposed theoretical method.
\end{abstract}

\section{Introduction}

In the last few years, the quadrotor aircraft has received extensive attention and application. Different from the fixedwing aircraft, there are some clear advantages for the quadrotor aircraft, such as simple structures, flexible operation, and vertical takeoff and landing [1]. Because of these characteristics, the quadrotor aircraft has good application scenarios in both military and civil fields, for example, environmental assessment and disaster relief [1-3].

With the continuous in-depth development of research on quadrotor aircraft, the problem of multiaircraft formation coordination control has attracted more and more attention because multiple aircraft can accomplish more complex tasks than the separate aircraft. Actually, the cooperative work of multiple aircraft means that not only more equipment can be loaded to adapt to more complex and precise working environments but also the efficiency and robustness of the entire system can be significantly improved. Nevertheless, since the quadrotor system is an underactuated system with strong coupling, nonlinearity, etc., it is difficult to design a controller for a single aircraft $[4,5]$, not to mention further coordinating the control of multiple aircraft on the basis of controlling one aircraft, which is even more challenging.

So far, the formation control has achieved a series of progress. Earlier studies $[6,7]$ realized the tracking control tasks for the time-varying formations. Based on the idea of distributed control, Lin et al. [8] proposed the formation control strategy about multiagent systems by using a complex Laplacian. Similarly for multiagent systems, considering the Lipschitz-type node dynamics, the consensus problem was studied by using the distributed method in [9]. Liu et al. [10] designed a distributed formation controller for mobile robots in consideration of sampling data and communication delay. For higher-order multileader multiagent systems, based on the observer and distributed method, Wen et al. [11] proposed a new class of containment protocols to weaken some irrational assumptions in the existing literature. For the coordinated control problem of mobile robots, Wang et al. [12] provided the corresponding solution by vision-based sensors. The global pinning synchronization problem was studied for a class of complex networks with switching directed topologies in [13]. Based on adaptive consensus theory and distributed method, a new kind of distributed dispatch algorithms were developed for smart grids subject to 
communication uncertainties in [14]. In addition, for the aircraft during the flight, the external disturbances are often unavoidable, such as wind disturbances. For the aircraft's attitude system, Wang and Su [15] investigated the dynamic surface control of the nonlinear transport aircraft model during the process of continuous heavy cargo airdrop in case of disturbance and actuator saturation. Chwa [16] proposed the fuzzy adaptive output feedback tracking control method for VTOL (vertical takeoff and landing) aircraft.

However, most of the existing results for formation controller algorithms only guarantee that the formation achievement is done with asymptotically stable. That is to say, the completion time of formation achievement will tend to be infinite. In practice, it is always hoped that the formation can be completed faster. To make up for the above shortcoming, the finite-time control method has been developed in [17-19] to complete the original intention of improving the convergence rate. As the name implies, the finite-time control means that, in a finite time, the states of the system will be stabilized to equilibrium. Combining with the nonlinear sliding mode control, Li et al. [20] proposed a finite-time formation control approach. For the spacecraft formation flying problem, Liu et al. [21] addressed the finite-time distributed orbit synchronization control strategy. Zhang et al. [22] realized the finite-time formation control for quadrotor aircraft with external disturbance. Although the finite-time control method has many advantages, its finite convergence time usually depends on the initial conditions. Considering this point, Polyakov [23] for the first time introduced the concept of fixed-time stability; in other words, the convergent time is independent of the initial conditions and can be predetermined. Wu et al. [24] considered the synchronization problem for a class of second-order master-slave nonlinear systems based on the fixed-time control theory and output feedback control method. Chu et al. [25] proposed the multirobot systems' formation tracking problem with nonholonomic constraints under the fixed-time theoretical framework. Gao and Guo [26] investigated a fixed-time formation control method for AUVs with leader-following control. For common second-order multiagent systems, Chu et al. [27] designed a robust fixed-time consensus controller.

Compared to the finite-time control method in [28], the fixed-time control has the advantage on the convergent time which is independent of the initial system states. Motivated by this consideration, this paper will study the formation control problem by using the fixed-time consensus algorithm. The main contribution of this paper is to realize the formation control of multiple aircraft with external disturbances in a fixed time. Firstly, the multiagent theory and the bilimit homogeneous theory are used to propose a fixed-time formation control algorithm. Then, the fixed-time attitude controller is designed for a single aircraft. At the same time, considering the negative influence of external disturbances on the aircraft, a fixed-time disturbance observer is designed for accurate/rapid observation of external disturbances. In this way, the external disturbances can be compensated by the observed values. Finally, the simulation results are provided to verify the dynamic- and steady-state performance of the system. Compared with the existing formation control algorithms, the main contribution of this paper is to propose a new fixed-time formation control algorithm in the presence of external disturbances, which can achieve attitude formation in a prescribed convergent time without depending on initial conditions. Hence, in an unknown environment, the proposed result in this paper can provide designer convergence information. To the best of the authors' knowledge, the presented results in this paper are novel in the literature.

\section{Prerequisite Knowledge}

2.1. Problem Description. The formation with $n$ four-rotor aircraft is considered, and let $\Gamma=\{1,2, \cdots, n\}$. From the viewpoint of modelling, the aircraft can usually use six variables to uniquely determine itself the position and attitude in three-dimensional space. Without loss of generality, in the inertial coordinate system, let $\Upsilon_{i}=\left(\eta_{i}, \iota_{i}, \kappa_{i}\right)^{T} \in$ $R^{3}$ be the aircraft's position and $\Theta_{i}=\left(p_{i}, q_{i}, r_{i}\right)^{T} \in R^{3}$ be the aircraft's attitude based on Euler angles. As a result, the coordinates of the aircraft are

$$
\left(\eta_{i}, \iota_{i}, \kappa_{i}, p_{i}, q_{i}, r_{i}\right)^{T} \in R^{6}, \quad i \in \Gamma .
$$

2.1.1. Position Dynamical Model. As that in [29, 30], the mathematical model of the position of each quadrotor can be described as

$$
\begin{aligned}
& \ddot{\eta}_{i}=\frac{T_{i}}{m_{i}}\left(\cos p_{i} \sin q_{i} \cos r_{i}+\sin p_{i} \sin r_{i}\right), \\
& \ddot{\imath}_{i}=\frac{T_{i}}{m_{i}}\left(\cos p_{i} \sin q_{i} \sin r_{i}-\sin p_{i} \cos r_{i}\right), \\
& \ddot{\kappa}_{i}=\frac{T_{i}}{m_{i}} \cos p_{i} \cos q_{i}-g, \quad i \in \Gamma,
\end{aligned}
$$

where $m_{i}$ denotes the $i$-th aircraft's mass, $T_{i}$ is the $i$-th aircraft's thrust, and $g$ is the gravitational acceleration which always exists.

2.1.2. Attitude Dynamical Model. By Euler angles, the attitude dynamical equation is given in [30]:

$$
\begin{aligned}
J_{i, 1} \ddot{p}_{i} & =l_{i} \tau_{i, 1}+d_{i, 1}(t), \\
J_{i, 2} \ddot{q}_{i} & =l_{i} \tau_{i, 2}+d_{i, 2}(t), \\
J_{i, 3} \ddot{r}_{i} & =c_{i} \tau_{i, 3}+d_{i, 3}(t), \quad i \in \Gamma,
\end{aligned}
$$

where $\tau_{i}=\left(\tau_{i, 1}, \tau_{i, 2}, \tau_{i, 3}\right)^{T}$ denotes the $i$-th aircraft's control torque, $c_{i}$ is the $i$-th aircraft's force-to-moment factor, $J_{i}=$ $\operatorname{diag}\left\{J_{i, 1}, J_{i, 2}, J_{i, 3}\right\}$ is the inertia matrix, and $l_{i}$ is the arm length of the $i$-th aircraft. In addition, the external disturbances are denoted by $d_{i}(t)=\left(d_{i, 1}(t), d_{i, 2}(t), d_{i, 3}(t)\right)^{T}$.

2.2. Control Objective. Let $\Upsilon_{d}=\left(\eta_{d}, l_{d}, \kappa_{d}\right)^{T}$ be the referenced formation trajectory. As we all know, in fact, the three-dimensional relative position between two points can be expressed in the form of a three-dimensional vector. The formation geometry in $3 \mathrm{D}$ space is given by vector 
$\Delta_{i j}=\left(\Delta \eta_{i j}, \Delta \iota_{i j}, \Delta \kappa_{i j}\right)^{T}, i, j \in \Gamma$. Hence, the aircraft $i$ and aircraft $j$ are hoped to have the relative positional deviation:

$$
\Delta_{i j}=\Delta_{i}-\Delta_{j}=\left(\Delta \eta_{i}, \Delta \iota_{i}, \Delta \kappa_{i}\right)^{T}-\left(\Delta \eta_{j}, \Delta \iota_{j}, \Delta \kappa_{j}\right)^{T},
$$

where $\Delta_{i}=\left(\Delta \eta_{i}, \Delta \iota_{i}, \Delta \kappa_{i}\right)^{T}$ and $\Delta_{j}=\left(\Delta \eta_{j}, \Delta \iota_{j}, \Delta \kappa_{j}\right)^{T}$ represent the coordinates of two points in the same three-dimensional rectangular coordinate system. Based on these assumption, the control objective is to design a controller such that there is a fixed time $T^{*}$ which is independent of any initial condition such that for any $i, j \in \Gamma$,

$$
\begin{aligned}
\lim _{t \longrightarrow T^{*}}\left[\begin{array}{c}
\eta_{i}(t)-\eta_{j}(t) \\
\iota_{i}(t)-\iota_{j}(t) \\
\kappa_{i}(t)-\kappa_{j}(t)
\end{array}\right]=\Delta_{i j}, \quad t<T^{*}, \\
\lim _{t \longrightarrow T^{*}} \frac{1}{n} \sum_{i=1}^{n}\left(\begin{array}{c}
\eta_{i}(t)-\eta_{j}(t) \\
\left.\iota_{i}(t)-\iota_{j}(t), \iota_{i}(t), \kappa_{i}(t)\right)^{T}=\left(\eta_{d}, \iota_{d}, \kappa_{d}\right)^{T}, \quad t<T^{*}, \\
\kappa_{i}(t)-\kappa_{j}(t)
\end{array}\right] \equiv \Delta_{i j}, \quad t \geq T^{*}, \\
\frac{1}{n} \sum_{i=1}^{n}\left(\eta_{i}(t), \iota_{i}(t), \kappa_{i}(t)\right)^{T} \equiv\left(\eta_{d}, \iota_{d}, \kappa_{d}\right)^{T}, \quad t \geq T^{*} .
\end{aligned}
$$

To solve the proposed problem, we made some assumptions.

Assumption 1. We assume the desired formation trajectory $\Upsilon_{d}=\left(\eta_{d}, \iota_{d}, \kappa_{d}\right)^{T}, \dot{\Upsilon}_{d}$, and $\ddot{\Upsilon}_{d}$ are bounded.

Assumption 2. For the external disturbance, we assume that $d_{i}(t)$ and $\dot{d}_{i}(t)$ are bounded. In other words, it can be found that there exist the known positive constants $L_{1}$ and $L_{2}$ such that $\left|d_{i}(t)\right| \leq L_{1}$ and $\left|\dot{d}_{i}(t)\right| \leq L_{2}$.

In order to realize the formation task, this paper intends to adopt multiagent theory. So next, we give some relevant general knowledge about graph theory.

2.3. Graph Theory. In this paper, the multiaircraft system with master-slave structure will be considered. Each aircraft can be seen as a node. The information interaction among $n$ nodes, i.e., $n$ follower agents, can be represented by the undirected graph $G(A)=\{V, E, A\} . V=\left\{v_{i}, i=1, \cdots, n\right\}$ is the set of nodes, $E \subseteq V \times V$ is the set of edges, and $A=\left[a_{i j}\right] \in R^{n \times n}$ is the weighted adjacency matrix of the graph $G(A)$ with nonnegative adjacency elements $a_{i j}$. If there is an edge from node $j$ to node $i$, i.e., $\left(v_{j}, v_{i}\right) \in E$, then $a_{i j}=a_{i j}>0$, which means there exists an available information channel from node $j$ to node $i$. The set of neighbors of node $i$ is denoted by $N_{i}=\left\{j:\left(v_{j}, v_{i}\right) \in E\right\}$. The out-degree of node $v_{i}$ is defined as $\operatorname{deg}_{\text {out }}\left(v_{i}\right)=d_{i}=$ $\sum_{j=1}^{n} a_{i j}=\sum_{j \in N_{i}} a_{i j}$. Then, the degree matrix of digraph $G$ is $D=\operatorname{diag}\left\{d_{1}, \cdots, d_{n}\right\}$, and the Laplacian matrix of digraph $G$ is $L=D-A$.

A path in graph $G$ from $v_{i_{1}}$ to $v_{i_{k}}$ is a sequence of $v_{i_{1}}, v_{i_{1}}$, $\cdots, v_{i_{k}}$ of finite nodes starting with $v_{i_{1}}$ and ending with $v_{i_{k}}$ such that $\left(v_{i_{l}}, v_{i_{l+1}}\right) \in E$ for $l=1,2, \cdots, k-1$. The graph $G$ is connected if there is a path between any two distinct vertices.

Assume that the reference state is represented by a leader. The connection weight between the $n$ agent and the leader is denoted by $b_{i}, i \in \Gamma$. If the $i$-th agent has access to the information of the leader, then $b_{i}>0$, otherwise, $b_{i}=0$. Let $B=\operatorname{diag}\left\{b_{1}, \cdots, b_{n}\right\}$.

Assumption 3. The graph for all follower agents is connected in the above communication topology. Meanwhile, make sure that at least one follower is directly connected to the leader to get the leader's information, i.e., $B \neq 0$.

2.4. Related Definitions and Lemmas. Next, some corresponding definitions and lemmas about fixed-time control theory are presented. First of all, we provide the relevant concepts of fixed-time stability.

Definition $1[18,23]$. Consider the nonlinear system.

$$
\begin{aligned}
\dot{x} & =f(x), \\
f(0) & =0, \quad x \in R^{n},
\end{aligned}
$$

where $f(\cdot): R^{n} \longrightarrow R^{n}$ is a continuous vector function. The origin is finite-time stable equilibrium if it is Lyapunov stable and finite-time convergence. The finite-time convergence means that there is a function $T\left(x_{0}\right)$ such that $\lim _{t \longrightarrow T\left(x_{0}\right)} x\left(t, x_{0}\right)=0$ and $x\left(t, x_{0}\right) \equiv 0, \forall t \geq T\left(x_{0}\right)$. The system is said to be fixed-time stable if it is finite-time stable and fixed-time convergence if the convergent time satisfies $\sup _{\forall x_{0}} T\left(x_{0}\right)<+\infty$.

Then, an important definition under the fixed-time theoretical framework is presented, i.e., the homogeneous definition.

Definition 2 [18]. For system (6), if for any $\varepsilon>0$, there exists $\left(r_{1}, \cdots, r_{n}\right)$ with $r_{i}>0, i=1, \cdots, n$ such that

$$
f_{i}\left(\varepsilon^{r_{1}} x_{1}, \cdots, \varepsilon^{r_{n}} x_{n}\right)=\varepsilon^{k+r_{i}} f_{i}(x), \quad i=1, \cdots, n,
$$

where $k \geq \min \left\{r_{i}, i=1, \cdots, n\right\}$, and then $f(x)$ is said to be homogeneous of degree $k$ with respect to the dilation $\left(r_{1}, \cdots, r_{n}\right)$.

Definition 3. Denote $\operatorname{sig}^{\alpha}(x)=\operatorname{sign}(x)|x|^{\alpha}$, where $\alpha \geq 0$, $x \in \mathscr{R}$, and $\operatorname{sign}(\cdot)$ is the standard sign function.

Finally, an important lemma for achieving fixed-time stability is presented.

Lemma 1 [31]. For system (6), suppose that $f(x)$ is a homogeneous vector field in the bilimit with associated triples $\left(r_{0}, k_{0}, f_{0}\right)$ and $\left(r_{\infty}, k_{\infty}, f_{\infty}\right)$. If the origins of systems 
$\dot{x}=f(x), \dot{x}_{0}=f_{0}(x)$, and $\dot{x}_{\infty}=f_{\infty}(x)$ are globally asymptotically stable, then the following statements hold:

(1) The origin of (6) is fixed-time stable when condition $k_{\infty}>0>k_{0}$ holds.

(2) Let $d_{V_{0}}$ and $d_{V_{\infty}}$ be the real numbers such that $d_{V_{0}}>\max _{1 \leq i \leq n} r_{0, i}$ and $d_{V_{\infty}}>\max _{1 \leq i \leq n} r_{\infty, i}$. There exists a continuous, positive definite, and proper function $V(x)$ such that the function $\partial V / \partial x_{i}$ is homogeneous in the bilimit with triples $\left(r_{0}, d_{V_{0}}-\right.$ $\left.r_{0, i}, \partial V_{0} / \partial x_{i}\right)$ and $\left(r_{\infty}, d_{V_{\infty}}-r_{\infty, i}, \partial V_{\infty} / \partial x_{i}\right)$, and the function $\left(\partial V / \partial x_{i}\right) f(x)$ is negative definite.

\section{Main Results}

In order to implement a fixed-time formation control algorithm, a two-step design procedure is applied, i.e., position and attitude control strategy design.

\subsection{Position Control Scheme Design. For the brevity, let}

$$
\begin{aligned}
& \mu_{i, 1}=\frac{T_{i}}{m_{i}}\left(\cos p_{i} \sin q_{i} \cos r_{i}+\sin p_{i} \sin r_{i}\right), \\
& \mu_{i, 2}=\frac{T_{i}}{m_{i}}\left(\cos p_{i} \sin q_{i} \sin r_{i}-\sin p_{i} \cos r_{i}\right), \\
& \mu_{i, 3}=\frac{T_{i}}{m_{i}} \cos p_{i} \cos q_{i}-g, \quad i \in \Gamma .
\end{aligned}
$$

(3) as

As a result, rewrite the position-loop dynamical equation

$$
\ddot{\eta}_{i}=\mu_{i, 1}, \ddot{l}_{i}=\mu_{i, 2}, \ddot{\kappa}_{i}=\mu_{i, 3} .
$$

Theorem 1. For the $i$-th aircraft's position system (9), if we design the following controller:

$$
\begin{aligned}
\mu_{i}= & -\sum_{j \in N_{i}} a_{i j}\left(\left[k_{p} \operatorname{sig}^{Q_{1}}\left(\Upsilon_{i}-\Upsilon_{j}-\Delta_{i j}\right)+k_{d} \operatorname{sig}^{Q_{2}}\left(\dot{\Upsilon}_{i}-\dot{\Upsilon}_{j}\right)\right]\right. \\
& \left.+\left[k_{p} \operatorname{sig}^{g_{1}^{\prime}}\left(\Upsilon_{i}-\Upsilon_{j}-\Delta_{i j}\right)+k_{d} \operatorname{sig}^{Q_{2}^{\prime}}\left(\dot{\Upsilon}_{i}-\dot{\Upsilon}_{j}\right)\right]\right) \\
& -b_{i}\left(\left[k_{p} \operatorname{sig}^{Q_{1}}\left(\Upsilon_{i}-\Upsilon_{d}-\Delta_{i}+\frac{1}{n} \sum_{i=1}^{n} \Delta_{i}\right)+k_{d} \operatorname{sig}^{Q_{2}}\left(\dot{\Upsilon}_{i}-\dot{\Upsilon}_{d}\right)\right]\right) \\
& +\left[k_{p} \operatorname{sig}^{g_{1}^{\prime}}\left(\Upsilon_{i}-\Upsilon_{d}-\Delta_{i}+\frac{1}{n} \sum_{i=1}^{n} \Delta_{i}\right)+k_{d} \operatorname{sig}^{g_{2}}\left(\dot{\Upsilon}_{i}-\dot{\Upsilon}_{d}\right)\right]+\ddot{\Upsilon}_{d},
\end{aligned}
$$

where $\mu_{i}=\left(\mu_{i, 1}, \mu_{i, 2}, \mu_{i, 3}\right)^{T}, 0<\varrho_{1}<1, \varrho_{2}=2 \varrho_{1} /\left(1+\varrho_{1}\right), \varrho_{1}^{\prime}>$ $1, \varrho_{2}^{\prime}=2 \varrho_{1}^{\prime} /\left(1+\varrho_{1}^{\prime}\right), k_{p}>0$, and $k_{d}>0$, and then the desired formation graphics can be completed in a fixed time.

Proof. Considering that the three-axis position model of aircraft is symmetrical, the form of their controllers is similar. Here, only the proof for $\eta$-axis is provided. First, we can perform a simple coordinate transformation to define the following error:

$$
e_{i, \eta}=\eta_{i}-\eta_{d}-\Delta \eta_{i}+\frac{1}{n} \sum_{i=1}^{n} \Delta \eta_{i}, \quad i \in \Gamma .
$$

Define the error vector as $x_{i, \eta}=\left[e_{i, \eta}, \dot{e}_{i, \eta}\right]^{T}$. Then, combining the position system (9) and the controller (1), the position error equation is

$$
\begin{aligned}
\dot{e}_{i, \eta}= & \dot{\eta}_{i}-\dot{\eta}_{d}, \\
\ddot{e}_{i, \eta}= & -\sum_{j \in N_{i}} a_{i j}\left(\left[k_{p} \operatorname{sig}^{Q_{1}}\left(e_{i, \eta}-e_{j, \eta}\right)+k_{d} \operatorname{sig}^{Q_{2}}\left(\dot{e}_{i, \eta}-\dot{e}_{j, \eta}\right)\right]\right. \\
& \left.+\left[k_{p} \operatorname{sig}^{Q^{\prime}}\left(e_{i, \eta}-e_{j, \eta}\right)+k_{d} \operatorname{sig}^{Q^{\prime}}\left(\dot{e}_{i, \eta}-\dot{e}_{j, \eta}\right)\right]\right) \\
& -b_{i}\left(\left[k_{p} \operatorname{sig}^{Q_{1}}\left(e_{i, \eta}\right)+k_{d} \operatorname{sig}^{Q_{2}}\left(\dot{e}_{i, \eta}\right)\right]+\left[k_{p} \operatorname{sig}^{g_{1}^{\prime}}\left(e_{i, \eta}\right)\right.\right. \\
& \left.\left.+k_{d} \operatorname{sig}^{Q_{2}^{\prime}}\left(\dot{e}_{i, \eta}\right)\right]\right), \quad i \in \Gamma .
\end{aligned}
$$

The next proof process is mainly based on Lemma 1 . First of all, we will show that the above error system (12) is globally asymptotically stable. Construct the following Lyapunov function as

$$
V=V_{1}+V_{2}
$$

where

$$
\begin{aligned}
& V_{1}=k_{p} \sum_{i=1}^{n}\left(\sum_{j=1}^{n} a_{i j} \int_{0}^{e_{i, \eta}-e_{j, \eta}} \operatorname{sig}^{Q_{1}}(\rho) d \rho+2 b_{i} \int_{0}^{e_{i, \eta}} \operatorname{sig}^{Q_{1}}(\rho) d \rho\right) \\
& +k_{p} \sum_{i=1}^{n}\left(\sum_{j=1}^{n} a_{i j} \int_{0}^{e_{i, \eta}-e_{j, \eta}} \operatorname{sig}^{Q_{1}^{\prime}}(\rho) d \rho+2 b_{i} \int_{0}^{e_{i, \eta}} \operatorname{sig}^{Q_{1}^{\prime}}(\rho) d \rho\right), \\
& V_{2}=\sum_{i=1}^{n} \dot{e}_{i, \eta}^{2}
\end{aligned}
$$

By calculation, the derivative of $V_{1}$ is

$$
\dot{V}_{1}=k_{p} \sum_{i=1}^{n}\left(\sum_{j=1}^{n} a_{i j} \operatorname{sig}^{Q_{1}}\left(e_{i, \eta}-e_{j, \eta}\right)\left(\dot{e}_{i, \eta}-\dot{e}_{j, \eta}\right)+2 b_{i} \operatorname{sig}^{Q_{1}}\left(e_{i, \eta}\right) \dot{e}_{i, \eta}\right)+k_{p} \sum_{i=1}^{n}\left(\sum_{j=1}^{n} a_{i j} \operatorname{sig}^{Q_{1}^{\prime}}\left(e_{i, \eta}-e_{j, \eta}\right)\left(\dot{e}_{i, \eta}-\dot{e}_{j, \eta}\right)+2 b_{i} \operatorname{sig}^{g_{1}^{\prime}}\left(e_{i, \eta}\right) \dot{e}_{i, \eta}\right) .
$$


Furthermore, we know the fact that $a_{i j}=a_{j i}, i, j \in \Gamma$. Meanwhile, it is not difficult to find that the function $\operatorname{sig}^{\alpha}(\cdot)$ is the odd function. Therefore, the derivative of $V_{2}$ can be written as

$$
\begin{aligned}
\dot{V}_{2}= & -k_{p} \sum_{i=1}^{n}\left(\sum_{j=1}^{n} a_{i j} \operatorname{sig}^{\varrho_{1}}\left(e_{i, \eta}-e_{j, \eta}\right)\left(\dot{e}_{i, \eta}-\dot{e}_{j, \eta}\right)+2 b_{i} \operatorname{sig}^{\varrho_{1}}\left(e_{i, \eta}\right) \dot{e}_{i, \eta}\right)-k_{p} \sum_{i=1}^{n}\left(\sum_{j=1}^{n} a_{i j} \operatorname{sig}^{\varrho_{1}^{\prime}}\left(e_{i, \eta}-e_{j, \eta}\right)\left(\dot{e}_{i, \eta}-\dot{e}_{j, \eta}\right)+2 b_{i} \operatorname{sig}^{\varrho_{1}^{\prime}}\left(e_{i, \eta}\right) \dot{e}_{i, \eta}\right) \\
& -k_{d} \sum_{i=1}^{n}\left(\sum_{j=1}^{n} a_{i j}\left|\dot{e}_{i, \eta}-\dot{e}_{j, \eta}\right|^{1+\varrho_{2}}+2 b_{i}\left|\dot{e}_{i, \eta}\right|^{1+\varrho_{2}}\right)-k_{d} \sum_{i=1}^{n}\left(\sum_{j=1}^{n} a_{i j}\left|\dot{e}_{i, \eta}-\dot{e}_{j, \eta}\right|^{1+\varrho_{2}^{\prime}}+2 b_{i}\left|\dot{e}_{i, \eta}\right|^{1+\varrho_{2}^{\prime}}\right) .
\end{aligned}
$$

Combined with the above derivatives of $V_{1}$ and $V_{2}$, it can be seen that the derivative of $V$ is

$$
\begin{aligned}
\dot{V}= & -k_{d} \sum_{i=1}^{n}\left(\sum_{j=1}^{n} a_{i j}\left|\dot{e}_{i, \eta}-\dot{e}_{j, \eta}\right|^{1+\varrho_{2}}+\sum_{j=1}^{n} a_{i j}\left|\dot{e}_{i, \eta}-\dot{e}_{j, \eta}\right|^{1+\varrho_{2}^{\prime}}\right. \\
& \left.+2 b_{i}\left|\dot{e}_{i, \eta}\right|^{1+\varrho_{2}}+2 b_{i}\left|\dot{e}_{i, \eta}\right|^{1+\varrho_{2}^{\prime}}\right) \leq 0 .
\end{aligned}
$$

Define set $\Xi=\left\{\left(e_{i, \eta}, \dot{e}_{i, \eta}\right) \mid \dot{V} \equiv 0\right\}$. It can be found that $\dot{V} \equiv 0$ implies $\dot{e}_{i, \eta} \equiv 0$ and $\ddot{e}_{i, \eta} \equiv 0$, which also implies that $e_{i, \eta} \equiv 0$ from (12), $\forall i \in \Gamma$. As a result of LaSalle's invariance principle [32], the system (12) is the globally asymptotically stable system.

Next, we explain that the closed-loop system (12) is homogeneous in the bilimit. Let $f_{s}\left(x_{i, \eta}\right)=\left[\dot{e}_{i, \eta}, \mu_{i, 1}-\ddot{\eta}_{d}\right]^{T}$. Then, the vectors

$$
\begin{aligned}
& f_{s_{0}}\left(x_{i, \eta}\right)=\left[\dot{e}_{i, \eta},-\sum_{j \in N_{i}} a_{i j}\left(\left[k_{p} \operatorname{sig}^{Q_{1}}\left(e_{i, \eta}-e_{j, \eta}\right)+k_{d} \operatorname{sig}^{Q_{2}}\left(\dot{e}_{i, \eta}-\dot{e}_{j, \eta}\right)\right]\right)-b_{i}\left[k_{p} \operatorname{sig}^{Q_{1}}\left(e_{i, \eta}\right)+k_{d} \operatorname{sig}^{Q_{2}}\left(\dot{e}_{i, \eta}\right)\right]\right]^{T},
\end{aligned}
$$

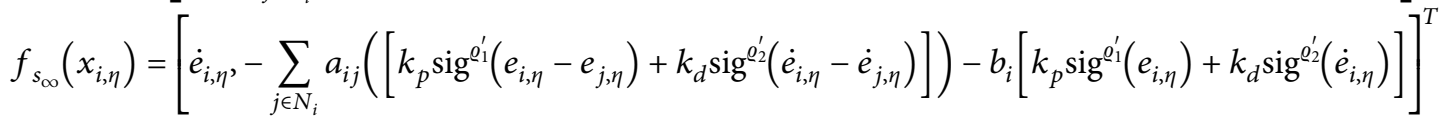

can be considered as approximating functions for $f_{s}$ in 0 limit and $\infty$ limit, respectively. According to Definition 2, i.e., the homogeneous system definition, it is obvious that the vector field $f_{s_{0}}$ is homogeneous with the degree of $k_{s_{0}}=\left(\varrho_{1}-1\right) / 2<0$ with respect to the dilation $r_{s_{0}}=$ $(\underbrace{s_{1}, \cdots, s_{1}}, \underbrace{s_{2}, \cdots, s_{2}})$, where $s_{1}=1$ and $s_{2}=\left(\varrho_{1}+1\right) / 2$. for $e_{1, \eta, \cdots, e_{n, \eta}}$ for $e_{1, \eta, \cdots, e_{n, \eta}}$

Similarly, the vector field $f_{s_{\infty}}$ is homogeneous, and its homogeneous degree is $k_{s_{\infty}}=\left(\varrho_{1}^{\prime}-1\right) / 2>0$ about the dilation $r_{s_{\infty}}=(\underbrace{s_{1}^{\prime}, \cdots, s_{1}^{\prime}}_{\text {for } e_{1, \eta}, \cdots, e_{n, \eta}}, \underbrace{s_{2}^{\prime}, \cdots, s_{2}^{\prime}}_{\text {for } \dot{e}_{1, \eta}, \cdots, \dot{e}_{n, \eta}})$, where $\quad s_{1}^{\prime}=1 \quad$ and $s_{2}^{\prime}=\left(\varrho_{1}^{\prime}+1\right) / 2$. Therefore, the closed-loop system $(12)$ is the bilimit homogeneous system with $\left(r_{s_{0}}, k_{s_{0}}, f_{s_{0}}\right)$ and $\left(r_{s_{\infty}}, k_{s_{\infty}}, f_{s_{\infty}}\right)$.

Finally, we will show that the systems $\dot{x}_{i, \eta}=f_{s_{0}}\left(x_{i, \eta}\right)$ and $\dot{x}_{i, \eta}=f_{s_{\infty}}\left(x_{i, \eta}\right)$ are globally asymptotically stable. For the system $\dot{x}_{i, \eta}=f_{s_{0}}\left(x_{i, \eta}\right)$, the Lyapunov function can be chosen as

$$
\begin{aligned}
V_{3}= & k_{p} \sum_{i=1}^{n}\left(\sum_{j=1}^{n} a_{i j} \int_{0}^{e_{i, \eta}-e_{j, \eta}} \operatorname{sig}^{\varrho_{1}}(\rho) d \rho+2 b_{i} \int_{0}^{e_{i, \eta}} \operatorname{sig}^{\varrho_{1}}(\rho) d \rho\right) \\
& +\sum_{i=1}^{n} \dot{e}_{i, \eta^{\prime}}^{2}
\end{aligned}
$$

Similar to the calculation for the above Lyapunov function $V$, the derivative of $V_{3}$ is

$$
\dot{V}_{3}=-k_{d} \sum_{i=1}^{n}\left(\sum_{j=1}^{n} a_{i j}\left|\dot{e}_{i, \eta}-\dot{e}_{j, \eta}\right|^{1+\varrho_{2}}+2 b_{i}\left|\dot{e}_{i, \eta}\right|^{1+\varrho_{2}}\right) \leq 0 \text {. }
$$

Similarly, for the system $\dot{x}_{i, \eta}=f_{s_{\infty}}\left(x_{i, \eta}\right)$, we choose

$$
\begin{aligned}
V_{4}= & k_{p} \sum_{i=1}^{n}\left(\sum_{j=1}^{n} a_{i j} \int_{0}^{e_{i, \eta}-e_{j, \eta}} \operatorname{sig}^{{ }^{\varrho^{\prime}}}(\rho) d \rho+2 b_{i} \int_{0}^{e_{i, \eta}} \operatorname{sig}^{\varrho_{1}^{\prime}}(\rho) d \rho\right) \\
& +\sum_{i=1}^{n} \dot{e}_{i, \eta}^{2},
\end{aligned}
$$

whose derivative is

$$
\dot{V}_{4}=-k_{d} \sum_{i=1}^{n}\left(\sum_{j=1}^{n} a_{i j}\left|\dot{e}_{i, \eta}-\dot{e}_{j, \eta}\right|^{1+\varrho_{2}^{\prime}}+2 b_{i}\left|\dot{e}_{i, \eta}\right|^{1+\varrho_{2}^{\prime}}\right) \leq 0 .
$$

The LaSalle's invariance principle ensures the global asymptotic stability of systems $\dot{x}_{i, \eta}=f_{s_{0}}\left(x_{i, \eta}\right)$ and $\dot{x}_{i, \eta}=$ $f_{s_{\infty}}\left(x_{i, \eta}\right)$.

In the end, based on Lemma 1, we can draw such a conclusion that the close-loop system (12) is globally fixed-time stable. Taking into account the previous definition of the error, it is obvious that multiple aircraft can accomplish position formation graphics in a fixed time. This completes the proof. 
3.2. Attitude Control Scheme Design. In the above design process of the position formation control scheme, using the idea of backstepping, the attitude information (i.e., Euler angle $\left.\Theta_{i}=\left(p_{i}, q_{i}, r_{i}\right)^{T}\right)$ of the aircraft is treated as a virtual control input. Next, the fixed-time attitude controller for each aircraft will be designed. First, in regard to each quadrotor aircraft, the desired attitude can be denoted as $\Theta_{i, d}=\left(p_{i, d}, q_{i, d}, r_{i, d}\right)^{T}$. According to (8), there is the following correspondence:

$$
\begin{aligned}
T_{i, d} & =m_{i} \sqrt{\mu_{i, \eta}^{2}+\mu_{i, l}^{2}+\left(\mu_{i, \kappa}+g\right)^{2}}, \\
p_{i, d} & =\arcsin \left(\frac{m_{i}}{T_{i, d}}\left(\mu_{i, \eta} \sin r_{i, d}-\mu_{i, l} \cos r_{i, d}\right)\right), \\
q_{i, d} & =\arctan \left(\frac{1}{\mu_{i, \kappa}+g}\left(\mu_{i, \eta} \cos r_{i, d}+\mu_{i, l} \sin r_{i, d}\right)\right), \quad i \in \Gamma .
\end{aligned}
$$

At the same time, it is worth noting that the desired yaw angle $r_{i, d}$ is an independent free variable. It can be set separately.

To remove the effect of external disturbances, there are many disturbance observer methods [33, 34]. Considering the influence of external disturbances on the aircraft, the corresponding fixed-time disturbance observers will be designed in the next part to achieve accurate observation of external disturbances within a fixed time. Define $d_{i}^{\prime}=\left(d_{i, 1}^{\prime}, d_{i, 2}^{\prime}, d_{i, 3}^{\prime}\right)^{T}=\left(d_{i, 1} / J_{i, 1}, d_{i, 2} / J_{i, 2}, d_{i, 3} / J_{i, 3}\right)^{T}$ as the new disturbance states. Meanwhile, define $\widehat{d}_{i}^{\prime}=\left(\widehat{d}_{i, 1}^{\prime}, \widehat{d}_{i, 2}^{\prime}, \widehat{d}_{i, 3}^{\prime}\right)^{T}=$ $\left(\widehat{d}_{i, 1} / J_{i, 1}, \widehat{d}_{i, 2} / J_{i, 2}, \widehat{d}_{i, 3} / J_{i, 3}\right)^{T}$ as the observers' states.

Lemma 2. Considering the attitude system (4) and Assumption 2, the states $\widehat{d}_{i}^{\prime}$ of the observers can be designed as

$$
\begin{aligned}
& \left\{\begin{array}{l}
\frac{\partial \widehat{\dot{p}}_{i}}{\partial t}=\frac{l_{i}}{J_{i, 1}} \tau_{i, 1}+\widehat{d}_{i, 1}^{\prime}+\rho_{1} \operatorname{sig}^{1 / 2}\left(\dot{p}_{i}-\widehat{\dot{p}}_{i}\right)+\rho_{2} \operatorname{sig}^{\alpha}\left(\dot{p}_{i}-\widehat{\dot{p}}_{i}\right), \\
\dot{\vec{d}}_{i, 1}^{\prime}=\rho_{3} \operatorname{sign}\left(\dot{p}_{i}-\widehat{\dot{p}}_{i}\right),
\end{array}\right. \\
& \left\{\begin{array}{l}
\frac{\partial \hat{\dot{q}}_{i}}{\partial t}=\frac{l_{i}}{J_{i, 2}} \tau_{i, 2}+\widehat{d}_{i, 2}^{\prime}+\rho_{1} \operatorname{sig}^{1 / 2}\left(\dot{q}_{i}-\widehat{\dot{q}}_{i}\right)+\rho_{2} \operatorname{sig}^{\alpha}\left(\dot{q}_{i}-\widehat{\dot{q}}_{i}\right), \\
\dot{\vec{d}}_{i, 2}^{\prime}=\rho_{3} \operatorname{sign}\left(\dot{q}_{i}-\hat{\dot{q}}_{i}\right), \\
\frac{\partial \hat{\dot{r}}_{i}}{\partial t}=\frac{c_{i}}{J_{i, 3}} \tau_{i, 3}+\hat{d}_{i, 3}^{\prime}+\rho_{1} \operatorname{sig}^{1 / 2}\left(\dot{r}_{i}-\hat{\dot{r}}_{i}\right)+\rho_{2} \operatorname{sig}^{\alpha}\left(\dot{r}_{i}-\hat{\dot{r}}_{i}\right), \\
\dot{\vec{d}}_{i, 3}^{\prime}=\rho_{3} \operatorname{sign}\left(\dot{r}_{i}-\hat{\dot{r}}_{i}\right),
\end{array}\right.
\end{aligned}
$$

where $\rho_{1}, \rho_{2}$, and $\rho_{3}$ are the gain parameters of the observers. Then, the estimated states will converge to the real states $d_{i}=$ $\left(d_{i, 1}, d_{i, 2}, d_{i, 3}\right)$ in a fixed time:

$$
T_{f} \leq\left(\frac{1}{\rho_{2}(\alpha-1) \varepsilon^{\alpha-1}}+\frac{2 \varepsilon^{1 / 2}}{\rho_{1}}\right)\left(1+\frac{1}{m\left(1 / M-h\left(\rho_{1}\right) / \rho_{1}\right)}\right),
$$

where $\varepsilon>0, \quad m=\rho_{3}-L_{2}, \quad M=\rho_{3}+L_{2}, \quad h\left(\rho_{1}\right)=1 / \rho_{1}+$ $\left(2 o / m \rho_{1}\right)^{1 / 3}$, and $o$ is the base of natural logarithms, provided that the following conditions hold for control gains: $\rho_{3}>L_{2}$ $h^{-1}\left(\rho_{1}\right) \rho_{1}>M$. The minimum value of $T_{f}(\varepsilon)$ is reached for $\varepsilon=\left(\rho_{1} / \rho_{2}\right)^{1 /(\alpha+1 / 2)}$.

Proof. First of all, let the corresponding disturbance observer error be $\sigma_{1}=\dot{p}_{i}-\widehat{\dot{p}}_{i}$ and $\sigma_{2}=d_{i, 1}^{\prime}-\widehat{d}_{i, 1}^{\prime}$. Then, the error dynamical equation can be obtained:

$$
\begin{aligned}
& \dot{\sigma}_{1}=\sigma_{2}-\rho_{1} \operatorname{sig}^{1 / 2}\left(\sigma_{1}\right)-\rho_{2} \operatorname{sig}^{\alpha}\left(\sigma_{1}\right), \\
& \dot{\sigma}_{2}=\dot{d}_{i, 1}^{\prime}-\rho_{3} \operatorname{sign}\left(\sigma_{1}\right) .
\end{aligned}
$$

On the basis of the result in [35], the system (26) is fixedtime stable, which means that the estimated state $\hat{d}_{i, 1}^{\prime}$ from the above observers will converge to the real state $d_{i, 1}^{\prime}$ in a fixed time. The proof is completed.

Since the disturbance observer mentioned above can accurately observe the disturbance in the aircraft's attitude system in a fixed time, a composite attitude controller based on disturbance compensation is presented to track the desired attitude.

Theorem 2. About each aircraft's attitude system (4), disturbance compensation (24) is added, and the attitude control algorithm is designed as 


$$
\begin{aligned}
& \tau_{i, 1}=\frac{J_{i, 1}}{l_{i}}\left(\ddot{p}_{i, d}+a_{p} \operatorname{sig}^{\beta_{1}}\left(p_{i, d}-p_{i}\right)+a_{d} \operatorname{sig}^{\beta_{2}}\left(\dot{p}_{i, d}-\dot{p}_{i}\right)+a_{p} \operatorname{sig}^{\beta_{1}^{\prime}}\left(p_{i, d}-p_{i}\right)+a_{d} \operatorname{sig}^{\beta_{2}^{\prime}}\left(\dot{p}_{i, d}-\dot{p}_{i}\right)-\widehat{d}_{i, 1}^{\prime}\right), \\
& \tau_{i, 2}=\frac{J_{i, 2}}{l_{i}}\left(\ddot{q}_{i, d}+a_{p} \operatorname{sig}^{\beta_{1}}\left(q_{i, d}-q_{i}\right)+a_{d} \operatorname{sig}^{\beta_{2}}\left(\dot{q}_{i, d}-\dot{q}_{i}\right)+a_{p} \operatorname{sig}^{\beta_{1}^{\prime}}\left(q_{i, d}-q_{i}\right)+a_{d} \operatorname{sig}^{\beta_{2}^{\prime}}\left(\dot{q}_{i, d}-\dot{q}_{i}\right)-\hat{d}_{i, 2}^{\prime}\right), \\
& \tau_{i, 3}=\frac{J_{i, 3}}{c_{i}}\left(\ddot{r}_{i, d}+a_{p} \operatorname{sig}^{\beta_{1}}\left(r_{i, d}-r_{i}\right)+a_{d} \operatorname{sig}^{\beta_{2}}\left(\dot{r}_{i, d}-\dot{r}_{i}\right)+a_{p} \operatorname{sig}^{\beta_{1}^{\prime}}\left(r_{i, d}-r_{i}\right)+a_{d} \operatorname{sig}^{\beta_{2}^{\prime}}\left(\dot{r}_{i, d}-\dot{r}_{i}\right)-\hat{d}_{i, 3}^{\prime}\right),
\end{aligned}
$$

where $0<\beta_{1}<1, \beta_{2}=2 \beta_{1} /\left(1+\beta_{1}\right), \beta_{1}^{\prime}>1, \beta_{2}^{\prime}=2 \beta_{1}^{\prime} /\left(1+\beta_{1}^{\prime}\right)$, $a_{p}>0, a_{d}>0$. So, in a fixed time, we can get the states $\left(p_{i}, q_{i}, r_{i}\right)^{T} \longrightarrow\left(p_{i, d}, q_{i, d}, r_{i, d}\right)^{T}$.

Proof. Firstly, considering the results of the previous observer (2), we can see that when $t \geq T_{f}$, there exists

$$
\begin{aligned}
& \widehat{d}_{i, 1}^{\prime}=d_{i, 1}^{\prime}, \\
& \hat{d}_{i, 2}^{\prime}=d_{i, 1}^{\prime}, \\
& \hat{d}_{i, 3}^{\prime}=d_{i, 3}^{\prime} .
\end{aligned}
$$

Let $e_{i, p}=p_{i, d}-p_{i}$ be the system error about roll angle $p_{i}$. When $t \geq T_{f}$, substituting the controller (27) into the attitude system (4), the attitude error system can be written as

$$
\begin{aligned}
\dot{e}_{i, p}= & \dot{p}_{i, d}-\dot{p}_{i}, \\
\ddot{e}_{i, p}= & -a_{p} \operatorname{sig}^{\beta_{1}}\left(e_{i, p}\right)-a_{d} \operatorname{sig}^{\beta_{2}}\left(\dot{e}_{i, p}\right)-a_{p} \operatorname{sig}^{\beta_{1}^{\prime}}\left(e_{i, p}\right) \\
& -a_{d} \operatorname{sig}^{\beta_{2}^{\prime}}\left(\dot{e}_{i, p}\right) .
\end{aligned}
$$

Obviously, the closed-loop system (31) and the system (12) have the similar structures. Hence, based on Lemma 1, the proof process is extremely similar, which is omitted here.

Remark 1. Note that the fixed-time control method is employed to design the position-loop controller, which implies that the desired Euler angles and rates may be nondifferentiable and nonsmooth. To this end, in order to obtain the differential signal for the attitude-loop controller design, here we can use the classical first-order differentiator (low-pass filter), i.e.:

$$
Y(s)=\frac{s}{T_{c} s+1} V(s)=\frac{1}{T_{c}}\left(1-\frac{1}{T_{c} s+1}\right) V(s),
$$

where $V(t)$ is the input signal, $Y(t)$ is the output signal, and $T_{c}$ is a proper small differential constant (in simulation, the time constant $T_{c}$ is chosen as $0.01 \mathrm{sec}$ ).

Remark 2. Note that in Theorem 1 and Theorem 2, the fixed-time stability analysis is provided for only the two subsystems (i.e., position-loop subsystem and attitude-loop subsystem) rather than the total cascaded system. Since the two subsystems are both fixed-time convergent, the stability analysis for the total cascaded system can be simplified. We just prove the state boundedness before the fixed time.

(1) For the attitude subsystem, before the time $T_{f}$, the attitude error system (29) is

$$
\begin{aligned}
\dot{e}_{i, p}= & \dot{p}_{i, d}-\dot{p}_{i}, \\
\ddot{e}_{i, p}= & -a_{p} \operatorname{sig}^{\beta_{1}}\left(e_{i, p}\right)-a_{d} \operatorname{sig}^{\beta_{2}}\left(\dot{e}_{i, p}\right) \\
& -a_{p} \operatorname{sig}^{\beta_{1}^{\prime}}\left(e_{i, p}\right)-a_{d} \operatorname{sig}^{\beta_{2}^{\prime}}\left(\dot{e}_{i, p}\right)-\left(\hat{d}_{i, 1}^{\prime}-d_{i, 1}^{\prime}\right) .
\end{aligned}
$$

To prove the system state is bounded in the time interval $\left[0, T_{f}\right]$, choose the Lyapunov function

$$
W=\frac{a_{p}}{1+\beta_{1}}\left|e_{i, p}\right|^{1+\beta_{1}}+\frac{a_{p}}{1+\beta_{1}^{\prime}}\left|e_{i, p}\right|^{1+\beta_{1}^{\prime}}+\frac{1}{2} \dot{e}_{i, p}^{2},
$$

which leads to

$$
\begin{aligned}
\dot{W}= & a_{p} \operatorname{sig}^{\beta_{1}}\left(e_{i, p}\right) \dot{e}_{i, p}+a_{p} \operatorname{sig}^{\beta_{1}^{\prime}}\left(e_{i, p}\right) \dot{e}_{i, p} \\
& +\dot{e}_{i, p}\left[-a_{p} \operatorname{sig}^{\beta_{1}}\left(e_{i, p}\right)-a_{d} \operatorname{sig}^{\beta_{2}}\left(\dot{e}_{i, p}\right)-a_{p} \operatorname{sig}^{\beta_{1}^{\prime}}\left(e_{i, p}\right)\right. \\
& \left.-a_{d} \operatorname{sig}^{\beta_{2}^{\prime}}\left(\dot{e}_{i, p}\right)-\left(\hat{d}_{i, 1}^{\prime}-d_{i, 1}^{\prime}\right)\right] \\
\leq & -\dot{e}_{i, p}\left(\hat{d}_{i, 1}^{\prime}-d_{i, 1}^{\prime}\right) \\
\leq & \frac{1}{2} \dot{e}_{i, p}^{2}+\frac{1}{2}\left(\hat{d}_{i, 1}^{\prime}-d_{i, 1}^{\prime}\right)^{2} .
\end{aligned}
$$

Since the term $\left(\hat{d}_{i, 1}^{\prime}-d_{i, 1}^{\prime}\right)$ is always bounded, there is a positive constant $C$ such that

$$
\dot{W} \leq C+W
$$

which implies

$$
W(t) \leq W(0) e^{t}+C t \leq W(0) e^{T_{f}}+C T_{f}, \quad \forall t \in\left[0, T_{f}\right] .
$$

Therefore, the attitude system's states are bounded for any time $t \in\left[0, T_{f}\right]$. 
(2) For the position system (2), it can be rewritten in the form of the cascaded system:

$$
\begin{aligned}
\ddot{\eta}_{i}= & \frac{T_{i}}{m_{i}}\left(\cos \left(p_{i, d}+p_{i}-p_{i, d}\right) \sin \left(q_{i, d}+q_{i}-q_{i, d}\right)\right. \\
& \cdot \cos \left(r_{i, d}+r_{i}-r_{i, d}\right)+\sin \left(p_{i, d}+p_{i}-p_{i, d}\right) \\
& \left.\cdot\left(r_{i, d}+r_{i}-r_{i, d}\right)\right) \\
= & \frac{T_{i}}{m_{i}}\left(\cos \left(p_{i, d}-e_{i, p}\right) \sin \left(q_{i, d}-e_{i, q}\right) \cos \left(r_{i, d}-e_{i, r}\right)\right. \\
& \left.+\sin \left(p_{i, d}-e_{i, p}\right) \sin \left(r_{i, d}-e_{i, r}\right)\right) .
\end{aligned}
$$

Under the proposed fixed-time attitude controller, the attitude tracking error $\left(e_{i, p}, e_{i, q}, e_{i, r}\right)$ will converge to zero in a fixed time $T^{*}$. After the time instant $T^{*}$, the position tracking error system (36) will be fixed-time convergence. By using a similar proof as that in the previous attitude control system, the position states are bounded during the time interval $\left[0, T^{*}\right]$.

\section{Numerical Simulations}

The formation task for a leader and three followers in aircraft is studied. Figure 1 shows the corresponding communication topology which is to be undirected connected graph. The weights are, respectively, given as $a_{12}=a_{21}=1, a_{23}=a_{32}=1$, and $b_{1}=1$. The desired formation graphics is set to a regular triangle on the $p$ - $q$ plane. According to Figure 1, the relative position deviation can be expressed as

$$
\begin{aligned}
\Delta \eta_{i} & =(0,1,0)^{T}, \\
\Delta \iota_{i} & =\left(\cos \left(\frac{5 \pi}{6}\right), \sin \left(\frac{-\pi}{6}\right), 0\right)^{T}, \\
\Delta \kappa_{i} & =\left(\cos \left(\frac{-\pi}{6}\right), \sin \left(\frac{-\pi}{6}\right), 0\right)^{T} .
\end{aligned}
$$

4.1. System and Controller Parameters. The virtual leader's position trajectory will be set as

$$
\left(\eta_{d}, \iota_{d}, \kappa_{d}\right)^{T}=(5 \times \sin (0.2 \times t), 5 \times \cos (0.2 \times t), 5)^{T} .
$$

In addition, the aircraft's system initial states are

$$
\begin{aligned}
& \left(\eta_{1}(0), \iota_{1}(0), \kappa_{1}(0), p_{1}(0), q_{1}(0), r_{1}(0)\right)=(-5,2,1,0,0,0), \\
& \left(\eta_{2}(0), \iota_{2}(0), \kappa_{2}(0), p_{2}(0), q_{2}(0), r_{2}(0)\right)=(2,-4,0,0,0,0), \\
& \left(\eta_{3}(0), \iota_{3}(0), \kappa_{3}(0), p_{3}(0), q_{3}(0), r_{3}(0)\right)=(1,3,0,0,0,0) .
\end{aligned}
$$

As that in Table 1, the system parameter values about some aircraft required in the simulation are provided.

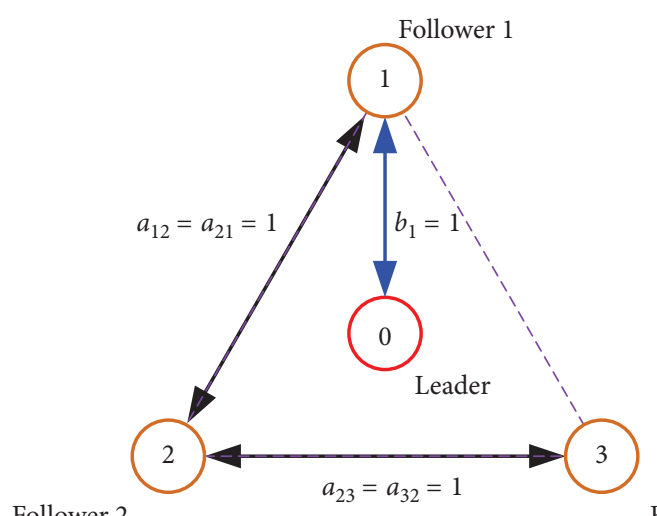

Follower 2

Follower 3

FIgURE 1: Referenced desired formation and the topology graph.

TABLE 1: $i$-th aircraft's system parameter values.

\begin{tabular}{lc}
\hline Descriptions & Parameter values \\
\hline $\begin{array}{l}\text { Inertia matrix } \\
\text { Force-to-moment } \\
\text { factor } \\
\begin{array}{l}\text { Mass } \\
\text { Arm length of the } \\
\text { aircraft }\end{array} \\
\begin{array}{l}\text { Gravitational } \\
\text { acceleration }\end{array} \\
\begin{array}{l}\text { Attitude-loop } \\
\text { disturbances }\end{array} \\
c_{i}=2.2 \\
m_{i}=0.468 \mathrm{~kg} \\
l_{i}=0.35 \mathrm{~m} \\
\\
\end{array} \quad\left\{\begin{array}{l}d_{i, 1}(t)=0.003 \sin (0.8 t+5) \\
d_{i, 2}(t)=0.002 \cos (1.5 t+3) \\
d_{i, 3}(t)=0.005 \sin (0.5 t+2), \quad i=1,2,3\end{array}\right.$ \\
\end{tabular}

Meanwhile, by Theorem 1 and Theorem 2, the control parameters can be set as $k_{p}=a_{p}=3.5, k_{d}=a_{d}=4.5$, $\alpha_{1}=\beta_{1}=3 / 4, \rho_{1}=6$, and $\rho_{2}=3$.

Through the fixed-time control scheme described in this paper, the flight trajectory curves of three aircraft formation in three-dimensional space are shown in Figure 2. Clearly, the proposed fixed-time control scheme can enable multiple aircraft to form a desired formation pattern within a fixed time and cause the entire aircraft formation to track the virtual leader's trajectory. In addition, the correlation curves of the aircraft's position and attitude are shown in Figures 3 and 4 . The response curves for the velocity and the angular velocity are, respectively, shown in Figures 5 and 6. As can be seen from Figure 2, under the fixed-time formation control scheme, the multiaircraft system with external disturbances can achieve better formation flying effect. Finally, in order to verify the statement that the convergence time is independent of initial state, Figure 7 shows the convergence time under different initial conditions, where $X(0)=\sum_{i=1}^{3}$ $\left(\left|\eta_{i}(0)-\eta_{d}(0)\right|+\left|\iota_{i}(0)-\iota_{d}(0)\right|+\left|\kappa_{i}(0)-\kappa_{d}(0)\right|\right)$. At the same time, the convergence time of the proposed method is compared with that of the finite-time control scheme in Figure 7. From Figure 7, it can be concluded that compared with the finite-time formation control scheme, the fixed-time formation control scheme has the better convergence performance, and the upper level of convergence time is independent of the initial state. 


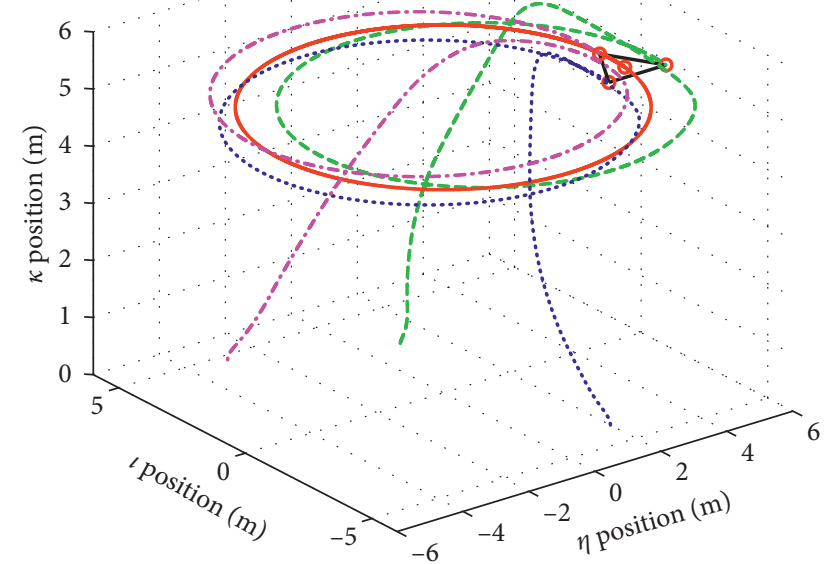

— Leader $\quad$....... Quadrotor 2

...- Quadrotor 1 --- Quadrotor 3

FIGURE 2: Formation flight path of multiple aircraft in 3D space.
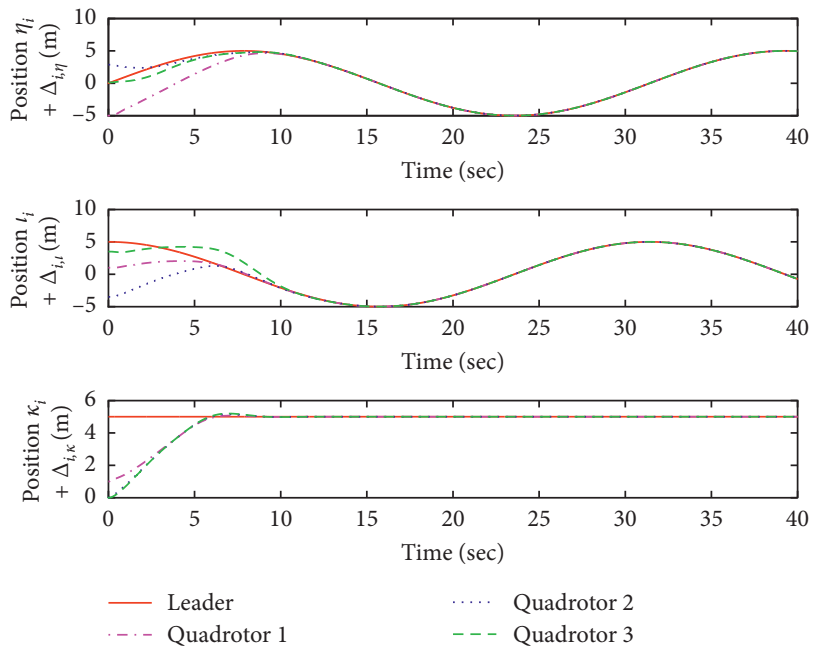

Figure 3: Position curves of aircraft.
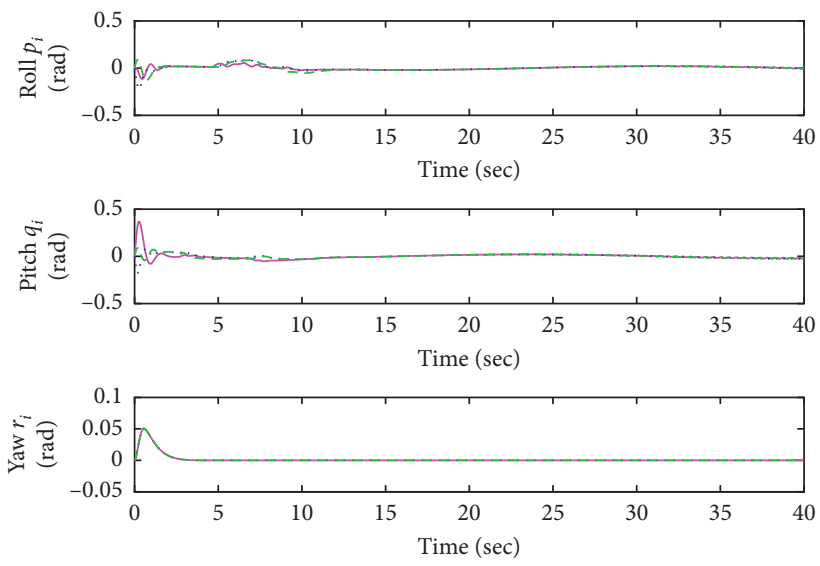

- Quadrotor 1

Quadrotor 2

Quadrotor 3

Figure 4: Attitude curves of aircraft.
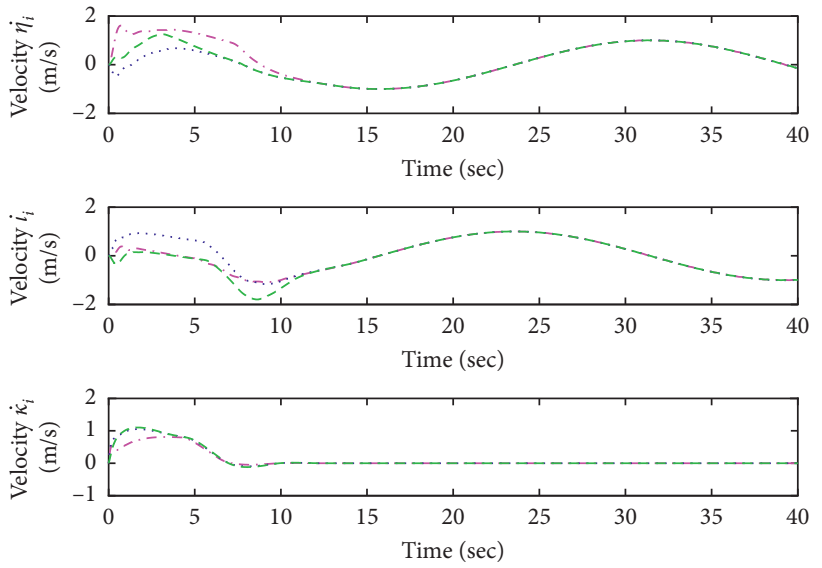

-.. Quadrotor 1

Quadrotor 2

- - - Quadrotor 3

FIgURE 5: Velocity curves of aircraft.
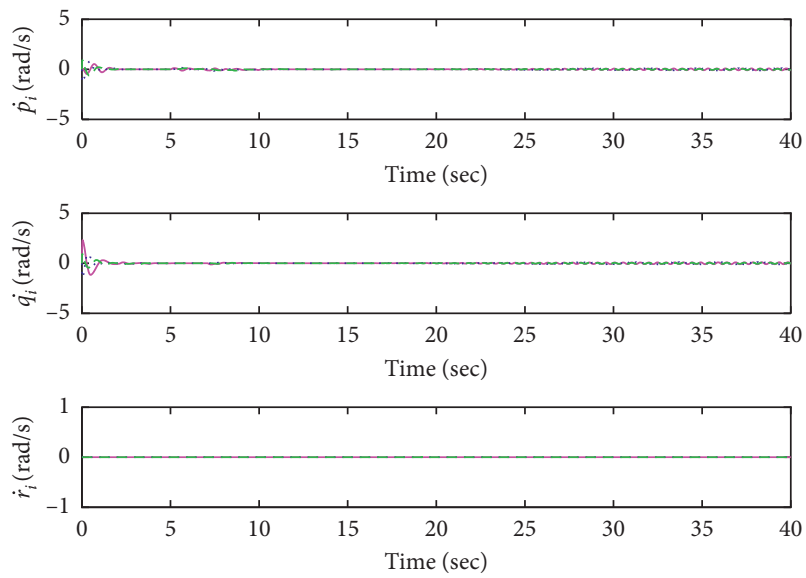

Quadrotor 1

Quadrotor 2

- Quadrotor 3

Figure 6: Angular velocity curves of aircraft.

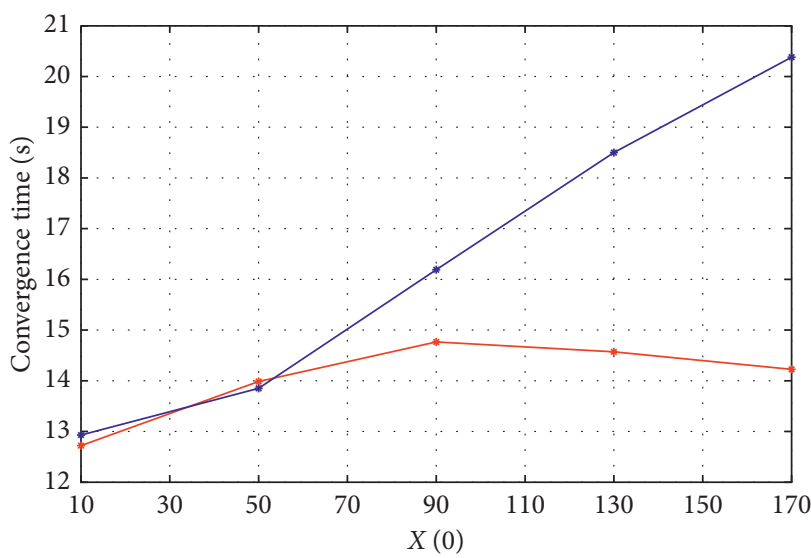

$\rightarrow$ Fixed-time control scheme

$\rightarrow$ Finite-time control scheme

Figure 7: Convergence time for the different initial conditions. 


\section{Conclusion}

To solve the formation control problem of a group of quadrotor aircraft, a distributed consensus algorithm has been proposed. To enhance the formation convergent rate, a fixed-time formation control scheme is proposed. Next, the external disturbance is introduced and a fixed-time disturbance observer is designed to compensate it. Some simulation results are presented to test the effectiveness of the proposed theoretical result. In future work, we will further study the directed connectivity of neighbor graphs and the antidisturbance control of aircraft.

\section{Data Availability}

No data were used to support this study.

\section{Conflicts of Interest}

The authors declare that there are no conflicts of interest regarding the publication of this paper.

\section{Acknowledgments}

This research was cosupported by the Guangdong Science and Technology Plan Project with research grants 2017A020208068 and 2016A020210123, Guangdong Natural and Science Foundation with research grant 2017A030310650, Common Technical Innovation Team of Guangdong Province on Preservation and Logistics of Agricultural Products with grant 2019KJ145, Guangzhou Science and Technology Plan Project with research grant 201704030131, and China Scholarship Council (CSC) grant.

\section{References}

[1] H. Lim, J. Park, D. Lee, and H. J. Kim, "Build your own quadrotor: open-source projects on unmanned aerial vehicles," IEEE Robotics \& Automation Magazine, vol. 19, no. 3, pp. 33-45, 2012.

[2] P. Castillo, R. Lozano, and A. Dzul, Modelling and Control of Mini-Flying Machines, Springer-Verlag, New York, NY, USA, 2005.

[3] A. Abdessameud and A. Tayebi, "Motion coordination for VTOL unmanned aerial vehicles," Advances in Industrial Control, Springer-Verlag, New York, NY, USA, 2013.

[4] Z. T. Dydek, A. M. Annaswamy, and E. Lavretsky, "Adaptive control of quadrotor UAVs: a design trade study with flight evaluations," IEEE Transactions on Control Systems Technology, vol. 21, no. 4, pp. 1400-1406, 2013.

[5] Y. Liu, Y. Fu, W. He, and Q. Hui, "Modeling and observerbased vibration control of a flexible spacecraft with external disturbances," IEEE Transactions on Industrial Electronics, vol. 66, no. 11, pp. 8648-8658, 2019.

[6] X. Dong, B. Yu, Z. Shi, and Y. Zhong, "Time-varying formation control for unmanned aerial vehicles: theories and applications," IEEE Transactions on Control Systems Technology, vol. 23, no. 1, pp. 340-348, 2015.

[7] X. Dong, Y. Zhou, Z. Ren, and Y. Zhong, “Time-varying formation tracking for second-order multi-agent systems subjected to switching topologies with application to quadrotor formation flying," IEEE Transactions on Industrial Electronics, vol. 64, no. 6, pp. 5014-5024, 2017.

[8] Z. Lin, L. Wang, Z. Han, and M. Fu, "Distributed formation control of multi-agent systems using complex Laplacian," IEEE Transactions on Automatic Control, vol. 59, no. 7, pp. 1765-1777, 2014.

[9] G. Wen, Z. Duan, G. Chen, and W. Yu, "Consensus tracking of multi-agent systems with Lipschitz-type node dynamics and switching topologies," IEEE Transactions on Circuits and Systems I: Regular Papers, vol. 61, no. 2, pp. 499-511, 2014.

[10] Z. Liu, W. Chen, J. Lu, H. Wang, and J. Wang, "Formation control of mobile robots using distributed controller with sampled-data and communication delays," IEEE Transactions on Control Systems Technology, vol. 24, no. 6, pp. 2125-2132, 2016.

[11] G. Wen, Y. Zhao, Z. Duan, W. Yu, and G. Chen, "Containment of higher-order multi-leader multi-agent systems: a dynamic output approach," IEEE Transactions on Automatic Control, vol. 61, no. 4, pp. 1135-1140, 2016.

[12] H. Wang, D. Guo, X. Liang, W. Chen, G. Hu, and K. K. Leang, "Adaptive vision-based leader-follower formation control of mobile robots," IEEE Transactions on Industrial Electronics, vol. 64, no. 4, pp. 2893-2902, 2017.

[13] G. Wen, W. Yu, G. Hu, J. Cao, and X. Yu, "Pinning synchronization of directed networks with switching topologies: a multiple Lyapunov functions approach," IEEE Transactions on Neural Networks and Learning Systems, vol. 26, no. 12, pp. 3239-3250, 2015.

[14] G. Wen, X. Yu, Z.-W. Liu, and W. Yu, “Adaptive consensusbased robust strategy for economic dispatch of smart grids subject to communication uncertainties," IEEE Transactions on Industrial Informatics, vol. 14, no. 6, pp. 2484-2496, 2018.

[15] L. Wang and J. Su, "Robust disturbance rejection control for attitude tracking of an aircraft," IEEE Transactions on Control Systems Technology, vol. 23, no. 6, pp. 2361-2368, 2015.

[16] D. Chwa, "Fuzzy adaptive output feedback tracking control of VTOL aircraft with uncertain input coupling and input-dependent disturbances," IEEE Transactions on Fuzzy Systems, vol. 23, no. 5, pp. 1505-1518, 2015.

[17] S. Bhat and D. S. Bernstein, "Finite-time stability of homogeneous systems," American Control Conference, vol. 4, no. 4, pp. 2513-2514, 1997.

[18] S. P. Bhat and D. S. Bernstein, "Finite-time stability of continuous autonomous systems," SIAM Journal on Control and Optimization, vol. 38, no. 3, pp. 751-766, 2000.

[19] Y. Cheng, H. Du, C. Yang, Z. Wang, J. Wang, and Y. He, "Fast adaptive finite-time voltage regulation control algorithm for a buck converter system," IEEE Transactions on Circuits and Systems II: Express Briefs, vol. 64, no. 9, pp. 1082-1086, 2017.

[20] T. Li, R. Zhao, C. L. P. Chen, L. Fang, and C. Liu, "Finite-time formation control of under-actuated ships using nonlinear sliding mode control," IEEE Transactions on Cybernetics, vol. 48, no. 11, pp. 3243-3253, 2018.

[21] R. Liu, X. Cao, and M. Liu, "Finite-time synchronization control of spacecraft formation with network-induced communication delay," IEEE Access, vol. 5, pp. 27242-27253, 2017.

[22] X. Zhang, J. Gao, W. Zhang, T. Zeng, and L. Ye, “distributed formation control for multiple quadrotor based on multiagent theory and disturbance observer," Mathematical Problems in Engineering, vol. 2019, pp. 1-11, 2019.

[23] A. Polyakov, "Nonlinear feedback design for fixed-time stabilization of linear control systems," IEEE Transactions on Automatic Control, vol. 57, no. 8, pp. 2106-2110, 2012. 
[24] D. Wu, H. Du, G. Wen, and J. Lü, "Fixed-time synchronization control for a class of master-slave systems based on homogeneous method," IEEE Transactions on Circuits and Systems II: Express Briefs, vol. 66, no. 9, pp. 1547-1551, 2019.

[25] X. Chu, Z. Peng, G. Wen, and A. Rahmani, "Distributed fixedtime formation tracking of multi-robot systems with nonholonomic constraints," Neurocomputing, vol. 313, pp. 167174, 2018.

[26] Z. Gao and G. Guo, "Fixed-time leader-follower formation control of autonomous underwater vehicles with event-triggered intermittent communications," IEEE Access, vol. 6, pp. 27902-27911, 2018.

[27] X. Chu, Z. Peng, G. Wen, and A. Rahmani, "Robust fixed-time consensus tracking with application to formation control of unicycles," IET Control Theory \& Applications, vol. 12, no. 1, pp. 53-59, 2018.

[28] H. Du, W. Zhu, G. Wen, Z. Duan, and J. Lu, "Distributed formation control of multiple quadrotor aircraft based on nonsmooth consensus algorithms," IEEE Transactions on Cybernetics, vol. 49, no. 1, pp. 342-353, 2019.

[29] B. Zhao, B. Xian, Y. Zhang, and X. Zhang, "Nonlinear robust adaptive tracking control of a quadrotor UAV via immersion and invariance methodology," IEEE Transactions on Industrial Electronics, vol. 62, no. 5, pp. 2891-2902, 2015.

[30] R. Xu and Ü. Özgüner, "Sliding mode control of a class of underactuated systems," Automatica, vol. 44, no. 1, pp. 233241, 2008.

[31] V. Andrieu, L. Praly, and A. Astolfi, "Homogeneous approximation, recursive observer design, and output feedback," SIAM Journal on Control \& Optimization, vol. 47, no. 4, pp. 1814-1850, 2009.

[32] H. K. Khalil, Nonlinear Systems, Prentice-Hall, Upper Saddle River, NJ, USA, 3rd edition, 2002.

[33] Y. Liu, F. Guo, X. He, and Q. Hui, "Boundary control for an axially moving system with input restriction based on disturbance observers," IEEE Transactions on Systems, Man, and Cybernetics: Systems, pp. 1-12, 2018.

[34] Y. Liu, Z. Zhao, and W. He, "Boundary control of an axially moving accelerated/decelerated belt system," International Journal of Robust and Nonlinear Control, vol. 26, no. 17, pp. 3849-3866, 2016.

[35] M. Basin, C. Bharath Panathula, and Y. Shtessel, "Multivariable continuous fixed-time second-order sliding mode control: design and convergence time estimation," IET Control Theory \& Applications, vol. 11, no. 8, pp. 1104-1111, 2017. 


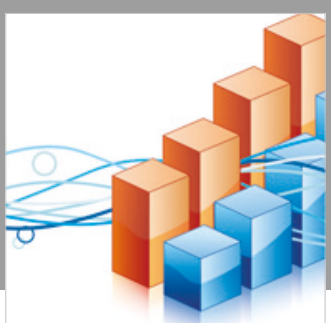

Advances in

Operations Research

\section{-n-m}
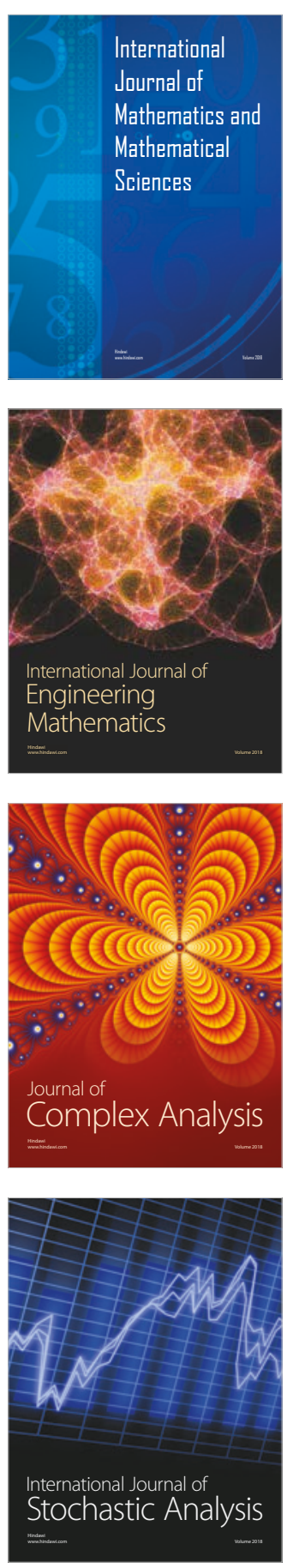
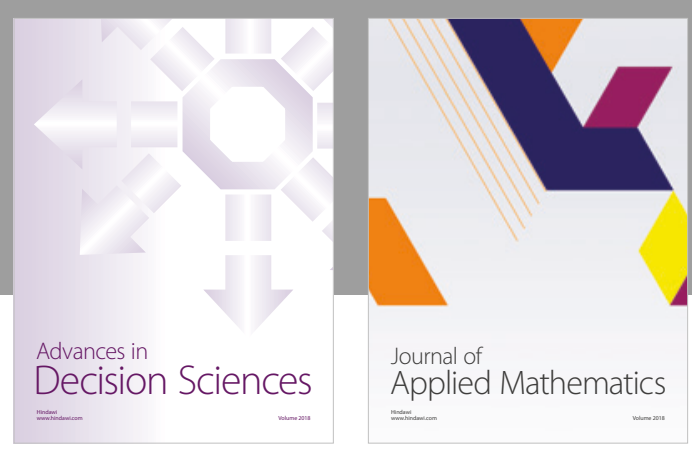

Journal of

Applied Mathematics
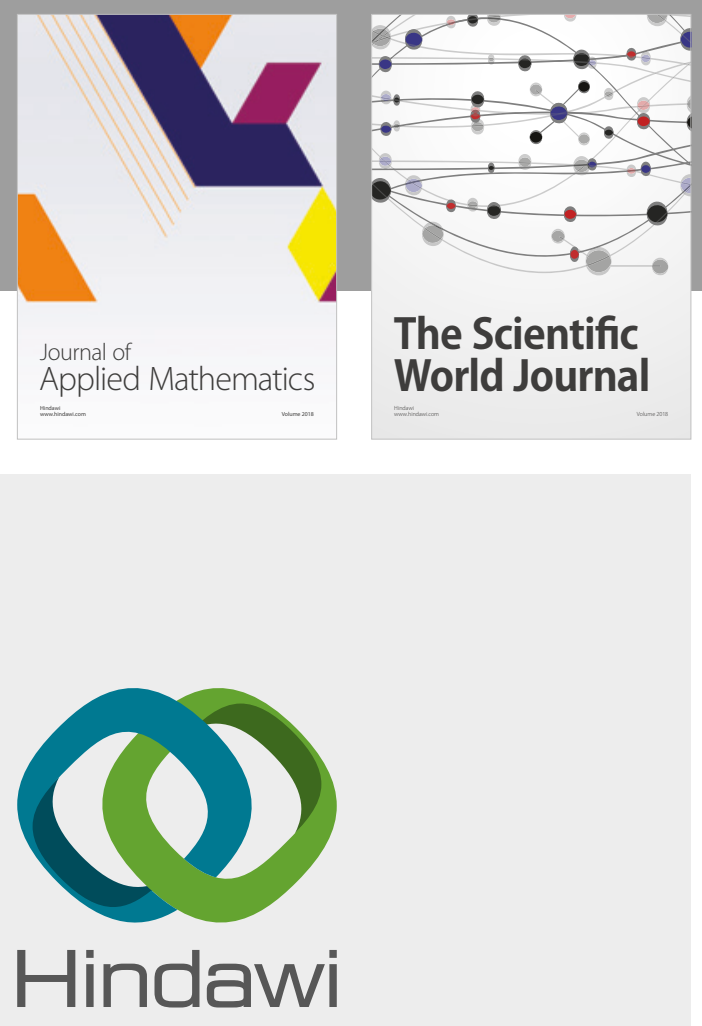

Submit your manuscripts at

www.hindawi.com

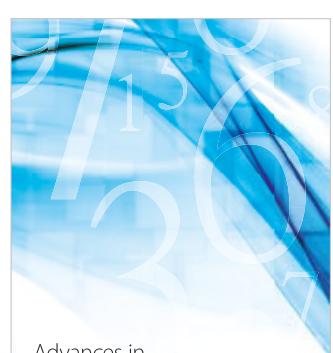

Advances in
Numerical Analysis
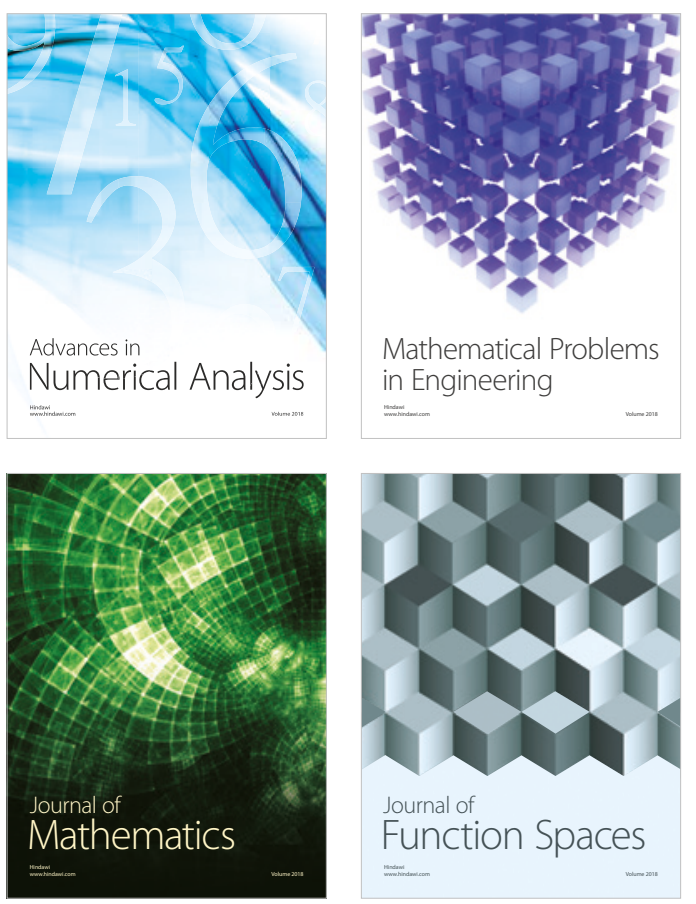

Mathematical Problems in Engineering

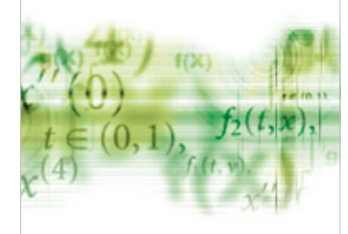

International Journal of

Differential Equations

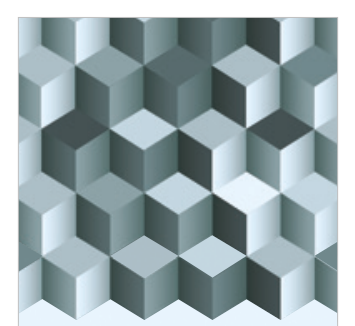

Journal of

Function Spaces

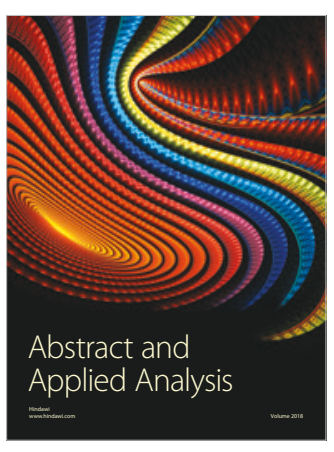

The Scientific

World Journal

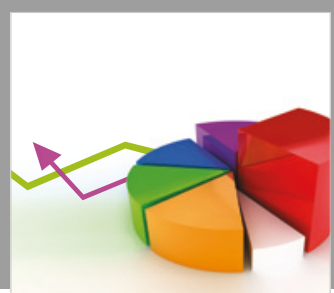

Journal of

Probability and Statistics
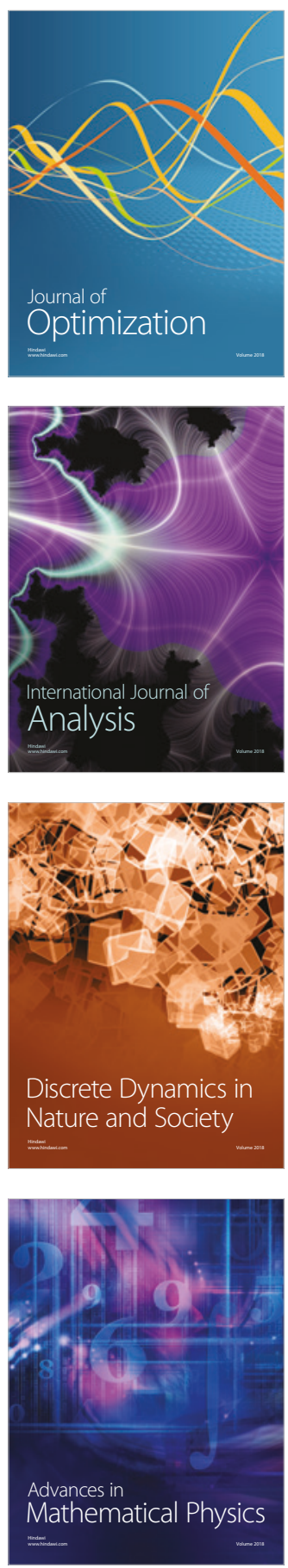